\title{
Expression of Fibronectin and Tenascin after Direct Capping of the Pulp with Mineral Trioxide Aggregate and Biodentine ${ }^{\circledR}$
}

\author{
Marina Eftimoska*, Vasilka Rendzova, Sonja Apostolska, Sasho Elencevski, Stevica Ristovska, Meri Pavlevska, \\ Vesna Filipovska-Micevska, Dusanka Stevanovic
}

Ss Cyril and Methodius University of Skopje, Faculty of Dentistry, Skopje, Republic of Macedonia

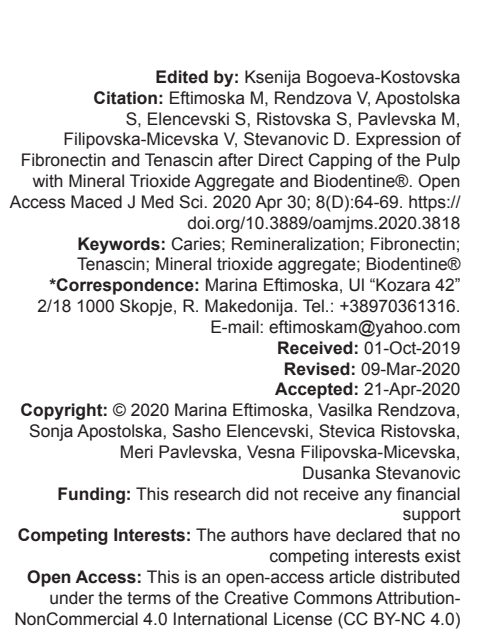

\section{Abstract}

BACKGROUND: Caries is a destructive process leading to progressive demineralization of the inorganic part of the tooth, accompanied by enzyme disintegration of the organic component of the tooth tissue. Considering the fact that caries activity can be stopped if the demineralization process is stopped, it is clear that the objectives of caries treatment are focused toward elimination of etiological factors and stimulation of regeneration of the dental tissues. That is why in the last years treatment of the caries disease is targeted to remineralization of the initial carious lesions, as well as on the biological behavior of the pulp-dentine complex after application of certain medications.

AIM: The aim of this study is to evaluate impact of the two materials for direct capping, mineral trioxide aggregate (MTA) and Biodentine ${ }$, on the expression of glycoproteins fibronectin (FN), and tenascin (TN), responsible for dentinogenesis.

METHODS: In the tests were used materials MTA and Biodentine, as agents for direct capping of pulp exposure. From 60 extracted teeth included in this in vitro study, tissue cuts were made. Each of them was then analyzed on a light microscope to determine the amount of two extracellular matrix glycoproteins, FN-C, and TN-C.

RESULTS: Our study show that there is an expressed immunoreactivity for FN and for TN in the fibronectin bridge under MTA and Biodentine $®$ after 8 , and even after 30 days of their application.

CONCLUSION: Based on this study, we can conclude that both materials - MTA and Biodentine - may induce reparative dentinogenesis, in which FN and TN have certainly a major role in the formation of the fibronectin matrix.

\section{Introduction}

Dental caries is a process that leads to a progressive demineralization of inorganic part of the tooth, accompanied by an enzyme disintegration of organic component of the tooth tissue. The carious lesion is initiated when dental plaque microorganisms (usually streptococcus mutans and Lactobacillus) ferment the carbohydrates and produce acids [1]. Therefore, it is considered that caries is primarily a bacterial disease, but it still has a multi-factorial etiology [2]. Caries activity can be stopped if demineralization process is stopped, and in other cases, it may spread more slowly, or to progress rapidly. Success of the therapy depends on the extent to which the pulp cell population can survive, as well as the ability of those cells to adequately respond to the carious lesion and to initiate appropriate reparatory process [3]. In recent years can be noticed a significant progress in the field of the caries disease treatment, which focuses on remineralization of the initial carious lesions.

One of the main roles of pulp tissue is physiological secretion of dentine throughout the entire life, as well as the ability to reinitiate dentinogenesis to protect it, in the event of external injuries. Direction of dentin tissue secretion depends on intensity of the initial stimulus. In general, reactive dentine is produced by the pre-existing odontoblasts, and reparative dentine is secreted by the newly differentiated odontoblastic cells [4].

The concept of vital pulp tissue therapy is based on the utilization of biological principles to maintain the pulp's vitality. Therefore, it is necessary not only to understand the pathophysiological processes occurring in the pulp-dentine complex but also to take into account the key factors contributing to success in the therapy (age of the patient, the absence of previous painful symptoms, a small pulpal exposure, minor pulpal hemorrhage, and a good pulp response to external stimuli) [5], [6], [7].

Nowadays, the clinicians use many materials and techniques for direct capping, including $\mathrm{Ca}(\mathrm{OH})_{2}$, hydrophilic resins, resin-modified glass ionomer cements, and tricalcium phosphates. More recently, mineral trioxide aggregate (MTA) is used, as well as bioactive silicate cement Biodentine, which stimulate odontoblasts and stimulate the defense 
mechanism of a pulp tissue. In cases of dentine tissue damage to a larger extent, when the pulp tissue is being exposed, newly differentiated cells are activated that secrete the reparative dentine and are responsible for formation of the dentinal bridging through pulp exposure. These cells in literature can be found as odontoblast such as cells, odontoblasts (new odontoblasts), or as the second generation of odontoblasts.

After the application of suitable material directly to the pulp exposure, the odontoblast like cells differentiate into dental-secrete cells and induce formation of a dentinal bridge within just few weeks of the application. The origin of these cells is not fully explained, but a group of authors believe that these processes resemble the processes involved in the growth of the tooth [8], [9].

Induction for differentiation of the odontoblast like cells is performed by bioactive molecules (growth factors, fibronectin [FN], tenascin [TN]), which originates from the circumpulpal dentine [10]. These molecules are considered to be the most important epigenetic signal that controls the cytological and functional differentiation of odontoblasts [11].

\section{Aim}

The goal of this study is to determine, with immunohistochemical tests, the level of glycoproteins $\mathrm{FN}$ and TN that play an important role in the process of odontogenesis.

\section{Materials and Methods}

\section{Materials}

We used materials MTA and Biodentine for the tests. The type of material that we examined, factory name, manufacturer, and chemical composition is given in Table 1.

Table 1: Materials included in the study

\begin{tabular}{|c|c|c|}
\hline Material & Manufacturer & Chemical composition \\
\hline $\begin{array}{l}\text { Mineral trioxide } \\
\text { aggregate }\end{array}$ & $\begin{array}{l}\text { Dentsply, Tulsa Dental } \\
\text { Products, Tulsa, OK, USA }\end{array}$ & Portland cement, bismuth oxide \\
\hline Biodentine & $\begin{array}{l}\text { Septodont, saint maur } \\
\text { des fosses, France }\end{array}$ & $\begin{array}{l}\text { Powder: Tricalcium silicate, calcium } \\
\text { carbonate, zirconium dioxide } \\
\text { Liquid: Calcium chloride, water reductor, water }\end{array}$ \\
\hline
\end{tabular}

\section{Methods}

To perform the above analyzes, representative samples of two tooth groups from patients aged 18-40 years were taken, in which a procedure of direct capping with appropriate material was carried out. A detailed medical history (anamnesis) was taken from the patients, followed by clinical evaluation, and intraoral radiographs for each tooth were made to determine absence of caries, trauma, periapical, and periodontal lesions.

The teeth that we set aside for these tests were vital and had an orthodontic indication for extraction and from the patient we provided consent to carry out these analyzes.

The pathohistological analysis took place at the Institute of Pathology in Skopje. The teeth included in this test had an indication for extraction for orthodontic purposes. In the selection of teeth from patients, the following criteria were included:

- $\quad$ The age of patients was from 18 to 30 years

- The patients did not have any systemic diseases

Teeth were with clinically normal pulp, without presence of caries and without any restoration There were no periodontal changes in the teeth.

This analyzes included 60 teeth, and depending on the material used for direct capping and duration of the same, they were divided into four groups:

1. Teeth with MTA - as a material for direct capping, which were extracted after 8 days of application of the material

2. Teeth with MTA - as a material for direct capping, which were extracted after 30 days of application of the material

3. Teeth with Biodentine - as a material for direct capping, which were extracted after 8 days of application of the material

4. Teeth with Biodentine - as a material for direct capping, which were extracted after 30 days of application of the material.

Patients had previously signed their agreement after it was explained to them for which purpose the extracted teeth would be used.

The procedure was started with anesthesia application (2\% Scandonest special; mepivacaine hydrochloride $35 \mathrm{mg}$ with $18 \mathrm{mcg}$ adrenaline), followed by preparation of the cavity with sterile diamond burs. (NTI Kahla GmbH 836KRS) The next step was trepanation with sterile round diamond bur (NTI 801L Kahla $\mathrm{GmbH}$ ) by constant cooling. After exposing of the pulp tissue, followed washing of the whole cavity with physiological solution. Then, we performed a hemostasis with sterile buffer soaked in physiological solution which we left there for 3-4 min until complete hemostasis was achieved. In the first and second group, we applied the MTA material (Dentsply, Tulsa Dental Products, Tulsa, OK, USA), prepared according to recommendations of the manufacturer, on the exposed part of the pulp, in a layer not thicker than $1.5 \mathrm{~mm}$. In the third and fourth groups, after preparing the Biodentine capsule, we applied the material in a layer of $1.5 \mathrm{~mm}$, directly onto the pulp. We then filled 
the cavities with glass ionomer cement (Securafil $囚$, Willmann and Pein $\mathrm{GmbH}$ ) and composite material SYNERGY®D6 (COLTENE®).

After 8 days, the teeth of the Groups 1 and 3 were extracted, and after 30 days the teeth of the second and fourth groups too. Then, we cut the teeth longitudinally and they were immersed in an agent for rapid de-mineralization of the dentine-osteomol, where they were left for 3-5 days, depending on whether the tissue cuts were ready to be made, with thickness of 5 $\mu \mathrm{m}$, and to be stained by the Brown-Brenn or Hemalumeosin method. These cuts were de-paraffinized in xylene, rehydrated in alcohol, and then washed in Tris-buffered saline (TBS). After finding of the trypsin antigens, the activity of endogenous peroxidase was blocked with $3 \% \mathrm{H}_{2} \mathrm{O}_{2}-5 \mathrm{~min}$. Then, the incisions were multiple incubated in various chemical solutions, and between each incubation they were washed twice with TBS. After the last incubation, there was a brown precipitate on the side of the antigens. Then, the preparations were stained with hematoxylin, then washed with water, dehydrated in ethanol and finally purified by xylene, and we monitored the reaction with development of a stained reaction with diaminobenzidine tetrachloride. Each of the tissue cuts was then analyzed on a light microscope, to determine the amount of the two extracellular matrix glycoproteins, FNC, and TNC. The amount of stained FN and TN was graded on a scale of I-IV.

I - specimens where there is no staining, II - specimens where there is a weak staining, III - specimens where the staining is medium, and IV severely stained.

All collected data that are relevant for preparation of the study were statistically processed (two-factor ANOVA), and the statistical series according to all defined variables are tabularly and graphically presented.

In immunohistochemical staining procedure, negative control staining was done in four intact molars by omitting exposure to the MTA and Biodentine. The expression of FNC and TNC in blood vessels was used as a positive internal control.

\section{Results}

Table 2 distribution of samples included in immunohistochemical analysis, where material for direct pulp capping was applied over the pulp exposition (a) MTA (b) Biodentine.

In immunohistochemical examination, tissue staining procedure showed lack of immunostaining in the negative control, that means no immunoreactivity for both FNC and TNC in the pulp, or in formed dentinal bridge.
Table 2: Distribution of samples included in imunohystochemical analysis, where material for direct pulp capping was applied over the pulp exposition a. MTA b. Biodentine

\begin{tabular}{llllll}
\hline Region & \multicolumn{2}{l}{ (a) First group } & & (b) Second group \\
\cline { 2 - 3 } \cline { 2 - 3 } & Mineral trioxide aggregate & Biodentine & \\
\cline { 2 - 5 } & $\mathrm{n}$ after 8 days & $\mathrm{n}$ after 30 days & $\mathrm{n}$ after 8 days & $\mathrm{n}$ after 30 days \\
\hline Premolars & 7 & 9 & 5 & 8 \\
Molars & 8 & 6 & 10 & 7 \\
Total & 15 & 15 & 15 & 15 \\
\hline
\end{tabular}

The expression of FNC and TNC in blood vessels was observed as a positive internal control.

The results of the immunohistochemical analyzes performed on 30 histological specimens where direct capping was performed with MTA and another 30 histological specimens where Biodentine was used as a material for direct capping, are showed in following tables and figures.

Each of the tissue cuts was then analyzed on a light microscope to determine the amount of the two extracellular matrix glycoproteins, FNC, and TNC. The amount of stained $\mathrm{FN}$ and $\mathrm{TN}$ is graded on a scale of I-IV.

\section{- $\quad \mathrm{I}$ - specimens where there is no staining \\ - $\quad$ II - specimens where there is a weak staining \\ - $\quad$ III - specimens where staining is medium and \\ - IV -severely stained.}

In the first group of teeth where direct capping was with MTA, after 8 days of the application, immunoreactivity is present both for FNC and for TNC in the dentinal bridge, and the expression of both glycoproteins is ranked from II - IV. Here, there is no statistically significant difference in the expression of FNC and TNC.

In the second group, after 30 days of the application, there is evident staining both for FNC and for TNC in the dentinal bridge, which indicates that their expression is present there. There is no statistically significant difference in this group too.

The third group of teeth were directly capped with Biodentine and after 8 days of the application there is medium to severe staining for FNC (III, IV) and a weak to medium staining for TNC (II, III) in the dentinal bridging. However, the statistical difference in the expression of both glycoproteins is insignificant.

In the teeth directly capped with Biodentine, even after 30 days of the application (fourth group), there is a staining for both FNC and TNC in the dentinal bridge, but in this group, there is also no statistically significant difference in the expression of FNC and TNC Table 3, Figures 1 and 2.

Table 3: Expression of FN and TN after application of MTA and Biodentine as direct pulp capping materials

\begin{tabular}{|c|c|c|c|c|c|c|c|c|}
\hline \multirow[t]{2}{*}{ Group } & \multicolumn{4}{|c|}{ Expression of fibronectin-C } & \multicolumn{4}{|c|}{ Expression of tenascin-C } \\
\hline & $\mathrm{I}$ & II & III & IV & $\mathrm{I}$ & II & III & $\mathrm{IV}$ \\
\hline MTA (after 8 days) & 0 & 3 & 9 & 3 & 0 & 3 & 9 & 3 \\
\hline MTA (after 30 days) & 0 & 9 & 3 & 3 & 0 & 9 & 6 & 0 \\
\hline Biodentine (after 8 days) & 0 & 0 & 10 & 5 & 0 & 6 & 9 & 0 \\
\hline Biodentine (after 30 days) & 0 & 9 & 6 & 0 & 0 & 11 & 4 & 0 \\
\hline
\end{tabular}




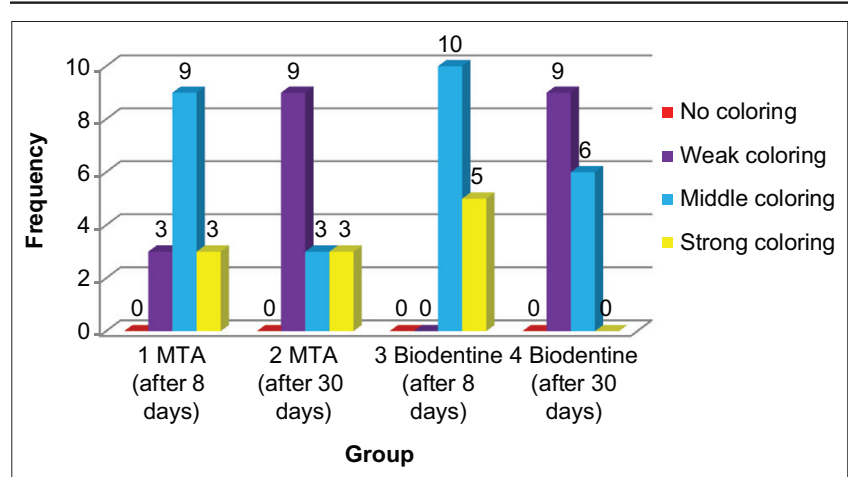

Figure 1: Expression of fibronectin

In comparing of the first and the second group (MTA after 8 and 30 days), there is a staining of FNC and TNC at both time intervals, there is however a decrease in the expression of the two glycoproteins in the dentinal bridge after 30 days (the second group). Reducing their expression is evident in the fourth group compared to the third, where capping is done with Biodentine. The difference in these cases is not statistically significant, Table 3, Figures 1 and 2.

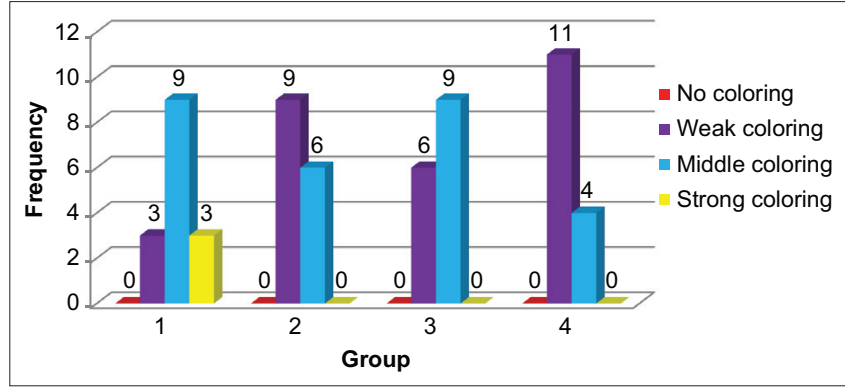

Figure 2: Expression of tenascin

When comparing the first group with the third group (MTA and Biodentine) after 8 days, a more intense staining of FNC is evident in the group of teeth capped with Biodentine (Figure 1), and slightly better staining of TNC in the group of teeth capped with MTA (Figure 2). The difference in the expression of the two glycoproteins in the dentinal bridge is not statistically significant between the two materials for direct capping.

After 30 days of direct capping, slightly more expressed staining is noticeable for both FNC and TNC in the dentinal bridge formed in the group of teeth capped with MTA (Group 2) compared to those where direct capping is performed with Biodentine (Group 4), but the difference is statistically insignificant, Table 3, Figures 1 and 2 .

\section{Discussion}

Immunohistochemical analyzes were done to evaluate the expression of FN and TN after direct capping of the pulp with MTA and with Biodentine $\AA$ after 8 and 30 days.
Throughout the present articles in the literature, exiting data show that a series of events taking place during reparative dentinogenesis resemble the processes observed during the primary dentinogenesis [12], [13]. There is no doubt that the role of extracellular matrix is very important, in which important biological processes take place, such as cellular migration, adhesion, proliferation, and differentiation [7], [14]. FN and TN are two noncollagen extracellular matrix proteins, which expression is evident during dentinogenesis. It is considered that they activate odontoblastic differentiation [11], [13], [15].

In the tertiary dentinogenesis, the matrix wherein $\mathrm{FN}$ is present is a reservoir of growing factors that are considered to be signaling molecules for differentiation of new odontoblasts [16], and also it is the medium where cell migration takes place. Pulpal cell adhesion to the matrix-that is rich with $F N$, is considered to have an important role in differentiating the cells responsible for creating solid tissue [15].

TN has a role in treating the wound and affects the cellular mobility. It can also modify the adhesion link between the cells and $\mathrm{FN}$, to allow easier cell migration through the matrix. Therefore, these two glycoproteins are considered to have an important role in the formation of new odontoblasts during tertiary dentinogenesis.

Tziafas et al. [17] point out that the high $\mathrm{pH}$ value of MTA allows release of dentine growth factors with which it is in contact, and they, on the other hand, is responsible for more intense dentinal bridging. They are also considered to have an impact on TN's expression [18].

Lukinmaa et al. [14] indicated that maintenance of the expression of FN and TN in human dental pulp in adults allows tertiary dentinogenesis to be carried out, whenever necessary.

In our study, there is an expressed immunoreactivity for $\mathrm{FN}$ and for $\mathrm{TN}$ in the fibronectin bridge under MTA and Biodentine $\AA$ after 8 , and even after 30 days of their application.

Fernandes et al. [19] in their study indicated the appearance of diffuse FN expression after pulp capping with calcium hydroxide after 7 and after 30 days of direct capping.

Piva et al. [20] indicated weak staining for FN and for TN after direct capping with calcium hydroxide after 1, 7, and 14 days from direct capping, and staining increases after 30 days of its application.

In our study, in comparing of the samples from the first and second groups (MTA after 8 and 30 days), there is a staining of FNC and TNC at both time intervals, there is however a decrease in the expression of the two glycoproteins in the dentinal bridge after 30 days (the second group), Figures 3 and 4. Reducing of their expression is evident in the fourth group compared to the third, where the capping is done with Biodentine. 


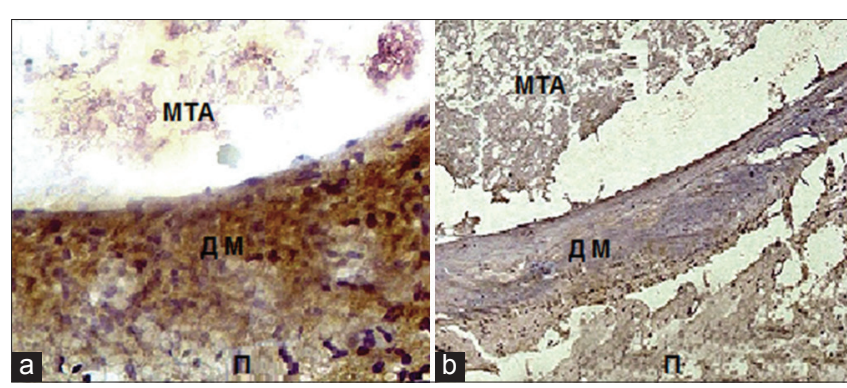

Figure 3: Immunohistochemical expression of fibronectin- $C$ in the matrix of the dental bridge - (a) 8 days after mineral trioxide aggregate (MTA) application as a material for direct pulp capping (III); (b) 30 days after MTA application as a material for direct pulp capping (II)

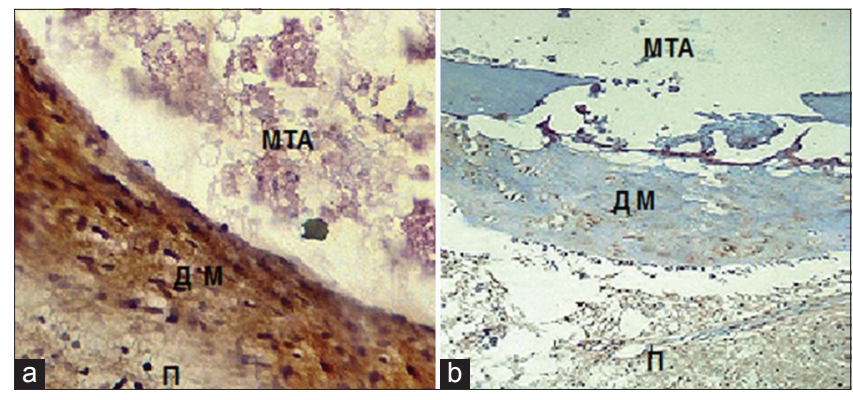

Figure 4: Immunohistochemical expression of tenascin- $C$ in the matrix of the dental bridge - (a) 8 days after mineral trioxide aggregate (MTA) application as a material for direct pulp capping (III); (b) 30 days after MTA application as a material for direct pulp capping (II)

In comparing of the first group with the third group (MTA and Biodentine) after 8 days, it is evident that there is a more intensive staining of FNC in the group of teeth capped with Biodentine, and slightly better staining of TNC in the group of teeth capped with MTA. The difference in the expression of the two glycoproteins in the dentinal bridge is not statistically significant between the two materials for direct capping, Figures 3 and 5.

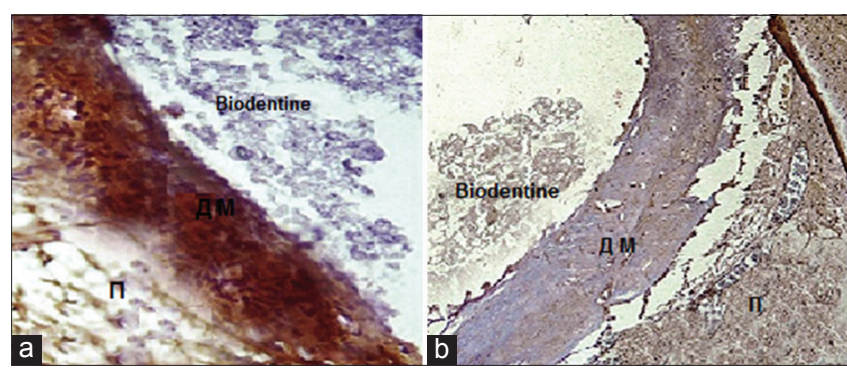

Figure 5: Immunohistochemical expression of fibronectin- $C$ in the matrix of the dental bridge - (a) 8 days after Biodentine application as a material for direct pulp capping (IV); (b) 30 days after Biodentine application as a material for direct pulp capping (II)

After 30 days of the direct capping, there is noticeably slightly more staining present, both for FNC and for TNC in the dentinal bridge formed in the group of teeth capped with MTA (Group 2) compared to those where direct capping is performed with Biodentine (Group 4), but the difference is statistically insignificant.

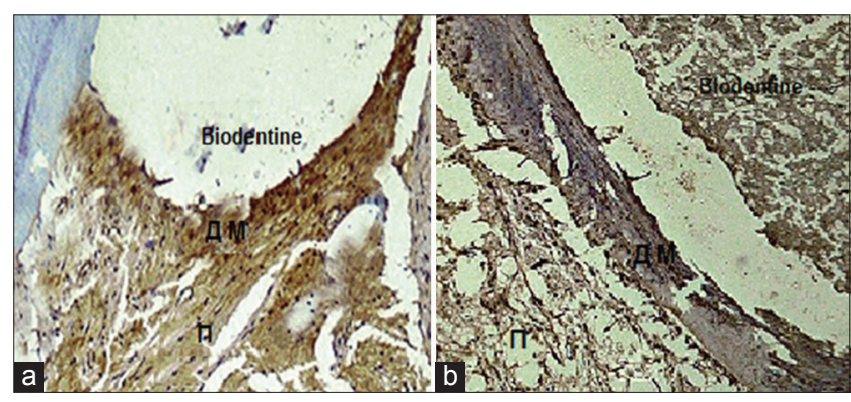

Figure 6: Immunohistochemical expression of tenascin-C in the matrix of the dental bridge - (a) 8 days after Biodentine application as a material for direct pulp capping (III); (b) 30 days after Biodentine application as a material for direct pulp capping (II)

The results we have obtained in our study are correlated with the fact that they play a major role in the fibronectin matrix, stimulating cell migration and differentiation, and their expression is reduced when the fibronectin matrix passes into a calcified bridge [20].

\section{Conclusion}

Based on the immunohistochemical studies, the results show that both materials - MTA and Biodentine - may induce reparative dentinogenesis, in which $\mathrm{FN}$ and $\mathrm{TN}$ have undoubtedly a major role in the formation of the fibronectin matrix, as well as cell migration and odontoblastic differentiation. Their expression is evident when both MTA and Biodentine are used as direct capping materials.

\section{References}

1. Dammaschkse TS. Anwendung eines neuen bioaktiven kalziumsilikat-zements zur direkten überkappung der pulpa. ZWR Das Dtsch Zahnärztl. 2012;121:360-4. https://doi. org/10.1055/s-0032-1327439

2. George B, Kim JS, Bakland LK. Direct pulp capping with minera trioxide aggregate. JADA. 2008;139(3):305-15. https://doi. org/10.14219/jada.archive.2008.0160

3. Jenny $\mathrm{N}$. Know about biocompatibility of dental materials: A review. Pyrex J Med Med Sci. 2017;4(5):33-43.

4. Meyer-Lueckel H, Paris S, Ekstrand KR, Effenberger S. Caries Management: Science and Clinical Practice. Part 1. New York: Stuttgart; 2013. https://doi.org/10.1055/b-0034-84421

5. Willershausen B, Willershausen I, Ross A, Velikonja S, Kasaj A Blettner M. Retrospective study on direct pulp capping with calcium hydroxide. Quintessence Int. 2011;42(2):165-71. https://doi.org/10.15517/ijds.v0i0.33920

PMid:21359251

6. Goldberg M, Pradelle-Plasse N, Tran XV, Colon P, Laurent $P$, Aubut $\mathrm{V}$, et al. Emerging trends in (bio) material research. In: Biocompatibility or Cytotoxic Effects of Dental Composites. 
Ch. 6. Brasil: Coxmoor Publishing Company, Working Group of ORE-FDI; 2009.

7. Goldberg M, Smith AJ. Cells and extracellular matrices of dentin and pulp: A biological basis for repair and tissue engineering. Crit Rev Oral Biol Med. 2004;15(1):13-27. https://doi. org/10.1177/154411130401500103

PMid: 14761897

8. Mitsiadis TA, Rahiotis C. Parallels between tooth development and repair: Conserved molecular mechanisms following carious and dental injury. J Dent Res. 2004;83(12):896-902. https://doi. org/10.1177/154405910408301202

PMid:15557394

9. Smith AJ, Lesot $\mathrm{H}$. Induction and regulation of crown dentinogenesis: Embryonic events as a template for dental tissue repair? Crit Rev Oral Biol Med. 2001;12(5):425-37. https://doi.org/10.1177/10454411010120050501 PMid:12002824

10. Gašić J, Rančić G, Radičević G, Radenković G. Molekularni Mehanizmi Indukcije Dentinogeneze. Niš: Sven; 2003. p. 9-95.

11. Ruch JV. Odontoblast differentiation and the formation of the odontoblast layer. J Dent Res. 1985;64:489-98. https://doi. org/10.1177/002203458506400402 PMid:3857251

12. Thesleff I, Mackie E, Vainio S, Chiquet-Ehrismann R. Changes in the distribution of tenascin during tooth development. Development. 1987;101(2):289-96.

PMid:2451586

13. Thesleff I, Vainio S, Jalkanen M. Cell matrix interactions in tooth development. Int J Dev Biol. 1989;33(1):91-7. PMid:2485706

14. Lukinmaa PL, Mackie EJ, Thesleff I. Immunohistochemical localization of the matrix glycoproteins-tenascin and the ED-sequence containing form of cellular fibronectin in human permanent teeth and periodontal ligament. J Dent Res. 1991;70(1):19-26. https://doi.org/10.1177/00220345910700010201
PMid: 1704020

15. Yoshiba N, Yoshiba K, Twaka M, Nakamura H, Osawa $H$. A confocal laser scanning microscopic study of the immunofluorescent localization of $\mathrm{FN}$ in the odontoblast layer of human teeth. Arch Oral Biol. 1994;39(5):395-400. https://doi. org/10.1016/0003-9969(94)90169-4 PMid:8060262

16. Tziafas D, Panagiotakopoulos N, Komnenou A Immunolocalization of fibronectin during the early response of dog dental pulp to demineralized dentin or calcium hydroxide containing cement. Arch Oral Biol. 1995;40:23-31. https://doi. org/10.1016/0003-9969(94)00148-5

PMid:7748109

17. Tziafas D,Pantelidou O,AlvanouA,Belibasakis G,PapadimitriouS The dentinogenic effect of mineral trioxide aggregate (MTA) in short-term capping experiments, Int Endod J. 2002;35(3):24554. https://doi.org/10.1046/j.1365-2591.2002.00471.x PMid: 11985676

18. Andelin WE, Shabahang S, WrightK, Torabinejad M. Identification of hard tissue after experimental pulp capping using dentin sialoprotein (DSP) as a marker. J Endod. 2003;29(10):646-50. https://doi.org/10.1097/00004770-200310000-00008 PMid: 14606787

19. Fernandes A, Silva G, Lopes N, Napimoga M, Benatti B, Alves J Direct capping of human pulps with a dent in bonding system and calcium hydroxide: an immunohistochemical analysis. Oral Surg Oral Med Oral Pathol Oral Radiol Endod. 2008;105(3): 385-90. https://doi.org/10.1016/j.tripleo.2007.08.031 PMid: 18280971

20. Piva E, Tarquinio S, Silva A, Araujo V. Immunohistochemical expression of fibronectin and tenascin after direct pulp capping with calcium hydroxide. Oral Surg Oral Med Oral Pathol Oral Radiol Endod. 2006;102(4):e66-71. https://doi.org/10.1016/j. tripleo.2006.01.015

PMid:16997097 